
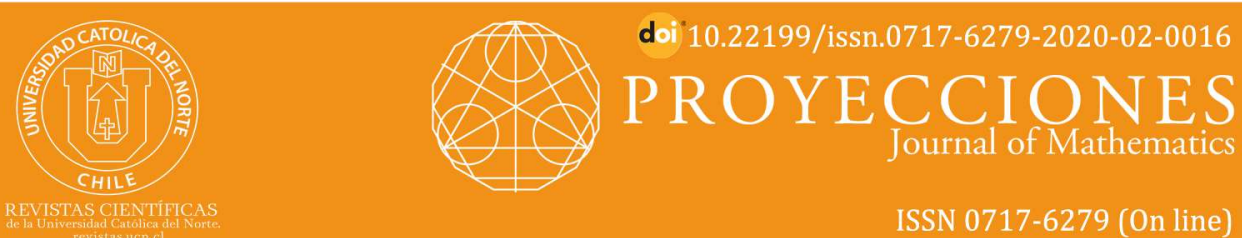

\title{
On rough convergence of triple sequence space of Bernstein operator of fuzzy numbers defined by a metric
}

M. Jeyaram Bharathi ${ }^{1}$

S. Velmurugan ${ }^{2}$ @ orcid.org/0000-0002-9749-4099

N. Subramanian ${ }^{3}$ (1) orcid.org/0000-0001-8096-742X

R. Srikanth ${ }^{4}$ @ orcid.org/0000-0003-1402-3599

Hindustan Institute of Technology and Science, Dept. of Mathematics, Chennai, TN, India.

1曰 jeyarambharathi@yahoo.com ; ${ }^{\square}$ ksvelmurugan.09@gmail.com

SASTRA University, Dept. of Mathematics, Thanjavur, TN, India.

3®nsmaths@gmail.com ; ${ }^{4}$ srikanth.sresam@gmail.com

\section{Received: February 2018 | Accepted: March 2018}

\section{Abstract:}

We define the concept of rough limit set of a triple sequence space of Bernstein polynomials of fuzzy numbers and obtain the relation between the set of rough limit and the extreme limit points of a triple sequence space of Bernstein polynomials of fuzzy numbers. Finally, we investigate some properties of the rough limit set of Bernstein polynomials.

Keywords: Triple sequences; Rough convergence; Closed and convex; Cluster points and rough limit points; Fuzzy numbers; Bernstein polynomials.

MSC (2010): 40F05, 40J05, 40G05.

\section{Cite this article as (IEEE citation style):}

M. Jeyaram Bharathi, S. Velmurugan, N. Subramanian, and R.

Srikanth, "On rough convergence of triple sequence space of

Bernstein operator of fuzzy numbers defined by a metric", Proyecciones (Antofagasta, On line), vol. 39, no. 2, pp. 261-274, Apr. 2020, doi: 10.22199/issn.0717-6279-2020-02-0016.

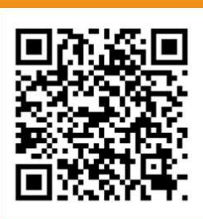

Article copyright: (C) 2020 M. Jeyaram Bharathi, S. Velmurugan, N. Subramanian, and R. Srikanth. This is an open access article distributed under the terms of the Creative Commons Licence, which permits unrestricted use and distribution provided the original author and source are credited. (cc) BY 


\section{Introduction}

The idea of rough convergence was first introduced by Phu [12-14] in finite dimensional normed spaces. He showed that the set $L I M_{x}^{r}$ is bounded, closed and convex; and he introduced the notion of rough Cauchy sequence. He also investigated the relations between rough convergence and other convergence types and the dependence of $L I M_{x}^{r}$ on the roughness of degree $r$.

Aytar [1] studied of rough statistical convergence and defined the set of rough statistical limit points of a sequence and obtained two statistical convergence criteria associated with this set and prove that this set is closed and convex. Also, Aytar [2] studied that the $r$ - limit set of the sequence is equal to intersection of these sets and that $r$ - core of the sequence is equal to the union of these sets. Dündar and Cakan [11] investigated of rough ideal convergence and defined the set of rough ideal limit points of a sequence The notion of $I$ - convergence of a triple sequence spaces which is based on the structure of the ideal $I$ of subsets of $\mathbf{N} \times \mathbf{N} \times \mathbf{N}$, where $\mathbf{N}$ is the set of all natural numbers, is a natural generalization of the notion of convergence and statistical convergence.

Let $K$ be a subset of the set of positive integers $\mathbf{N} \times \mathbf{N} \times \mathbf{N}$ and let us denote the set $K_{i k \ell}=\{(m, n, k) \in K: m \geq i, n \leq j, k \leq \ell\}$. Then the natural density of $K$ is given by

$$
\delta(K)=\lim _{i, j, \ell \rightarrow \infty} \frac{\left|K_{i j \ell}\right|}{i j \ell},
$$

where $\left|K_{i j \ell}\right|$ denotes the number of elements in $K_{i j \ell}$.

The Bernstein operator of order $(r, s, t)$ is given by

$B_{r s t}(f, x)=\sum_{m=0}^{r} \sum_{n=0}^{s} \sum_{k=0}^{t} f\left(\frac{m n k}{r s t}\right)(\stackrel{r}{m})\left(\begin{array}{l}s \\ n\end{array}\right)\left(\begin{array}{l}t \\ k\end{array}\right) x^{m+n+k}(1-x)^{(m-r)+(n-s)+(k-t)}$ where $f$ is a continuous (real or complex valued) function defined on $[0,1]$.

Throughout the paper, $\mathbf{R}$ denotes the real of three dimensional space with metric $(X, d)$. Consider a triple sequence of Bernstein polynomials $\left(B_{m n k}(f, x)\right)$ such that $\left(B_{m n k}(f, x)\right) \in \mathbf{R}, m, n, k \in \mathbf{N}$.

Let $f$ be a continuous function defined on the closed interval $[0,1]$. A triple sequence of Bernstein polynomials $\left(B_{m n k}(f, x)\right)$ is said to be statistically convergent to $0 \in \mathbf{R}$, written as $s t-\lim x=0$, provided that the set

$$
K_{\epsilon}:=\left\{(m, n, k) \in \mathbf{N}^{3}:\left|B_{m n k}(f, x)-f(x)\right| \geq \epsilon\right\}
$$


has natural density zero for any $\epsilon>0$. In this case, 0 is called the statistical limit of the triple sequence of Bernstein polynomials. i.e., $\delta\left(K_{\epsilon}\right)=0$. That is,

$$
\lim _{r s t \rightarrow \infty} \frac{1}{r s t}\left|\left\{(m, n, k) \leq(r, s, t):\left|B_{m n k}(f, x)-(f, x)\right| \geq \epsilon\right\}\right|=0 .
$$
$f(x)$.

In this case, we write $\delta-\lim B_{m n k}(f, x)=f(x)$ or $B_{m n k}(f, x) \rightarrow S_{B}$

Throughout the paper, $\mathbf{N}$ denotes the set of all positive integers,$\chi_{A^{-}}-$ the characteristic function of $A \subset \mathbf{N}, \mathbf{R}$ the set of all real numbers. A subset $A$ of $\mathbf{N}$ is said to have asymptotic density $d(A)$ if

$$
d(A)=\lim _{i j \ell \rightarrow \infty} \frac{1}{i j \ell} \sum_{m=1}^{i} \sum_{n=1}^{j} \sum_{k=1}^{\ell} \chi_{A}(K) .
$$

A triple sequence (real or complex) can be defined as a function $x$ : $\mathbf{N} \times \mathbf{N} \times \mathbf{N} \rightarrow \mathbf{R}(\mathbf{C})$, where $\mathbf{N}, \mathbf{R}$ and $\mathbf{C}$ denote the set of natural numbers, real numbers and complex numbers respectively. The different types of notions of triple sequence was introduced and investigated at the initial by Sahiner et al. [15,16], Esi et al. [3-8], Datta et al. [9], Subramanian et al. [17], Debnath et al. [10] and many others.

A triple sequence $x=\left(x_{m n k}\right)$ is said to be triple analytic if

$$
\sup _{m, n, k}\left|x_{m n k}\right|^{\frac{1}{m+n+k}}<\infty
$$

The space of all triple analytic sequences are usually denoted by $\Lambda^{3}$.

The set of fuzzy real numbers is denoted by $f(x)(\mathbf{R})$, and $d$ denotes the supremum metric on $f(X)\left(\mathbf{R}^{3}\right)$. Now let $r$ be nonnegative real number. A triple sequence space of Bernstein polynomials of $\left(B_{m n k}(f, X)\right)$ of fuzzy numbers is $r$ - convergent to a fuzzy number $f(X)$ and we write

$$
B_{m n k}(f, X) \rightarrow^{r} f(X) \text { as } m, n, k \rightarrow \infty,
$$

provided that for every $\epsilon>0$ there is an integer $m_{\epsilon}, n_{\epsilon}, k_{\epsilon}$ so that

$$
d\left(B_{m n k}(f, X), f(X)\right)<r+\epsilon \text { whenever } m \geq m_{\epsilon}, n \geq n_{\epsilon}, k \geq k_{\epsilon} .
$$


264 M. J. Bharathi, S. Velmurugan, N. Subramanian and R. Srikanth

The set $L I M^{r} B_{m n k}(f, X):=\left\{f(X) \in f(X)\left(\mathbf{R}^{3}\right): B_{m n k}(f, X) \rightarrow^{r} f(X)\right.$, as $\left.m, n, k \rightarrow \infty\right\}$ is called the $r$ - limit set of the triple sequence space of Bernstein polynomials of $\left(B_{m n k}(f, X)\right)$.

A triple sequence space of Bernstein polynomials of fuzzy numbers which is divergent can be convergent with a certain roughness degree. For instance, let us define

$$
B_{m n k}(f, X)=\left\{\begin{array}{lc}
\eta(X), & \text { if }(m, n, k) \text { is odd integer }, \\
\mu(X), & \text { otherwise }
\end{array}\right\}
$$

where

$$
\eta(X)=\left\{\begin{array}{cc}
X, & \text { if } X \in[0,1] \\
-X+2, & \text { if } X \in[1,2], \\
0, & \text { otherwise }
\end{array}\right\}
$$

and

$$
\mu(X)=\left\{\begin{array}{cc}
X-3, & \text { if } X \in[3,4] \\
-X+5, & \text { if } X \in[4,5], \\
0, & \text { otherwise }
\end{array}\right\}
$$

Then we have where

$$
L I M^{r} B_{m n k}(f, X)=\left\{\begin{array}{cc}
\phi, & \text { if } r<\frac{3}{2}, \\
{\left[\mu-r_{1}, \eta+r_{1}\right],} & \text { otherwise }
\end{array}\right\},
$$

where $r_{1}$ is nonnegative real number with $\left[\mu-r_{1}, \eta+r_{1}\right]:=\left\{B_{m n k}(f, X) \in f(X)\left(\mathbf{R}^{3}\right): \mu-r_{1} \leq B_{m n k}(f, X) \leq \eta+r_{1}\right\}$.

The ideal of rough convergence of a triple sequence space of Bernstein polynomials can be interpreted as follows:

Let $\left(B_{m n k}(f, Y)\right)$ be a convergent triple sequence space of Bernstein polynomials of fuzzy numbers. Assume that $\left(B_{m n k}(f, Y)\right)$ cannot be determined exactly for every $(m, n, k) \in \mathbf{N}^{3}$. That is, $\left(B_{m n k}(f, Y)\right)$ cannot be calculated so we can use approximate value of $\left(B_{m n k}(f, Y)\right)$ for simplicity of calculation. We only know that $\left(B_{m n k}(f, Y)\right) \in\left[\mu_{m n k}, \lambda_{m n k}\right]$, where $d\left(\mu_{m n k}, \lambda_{m n k}\right) \leq r$ for every $(m, n, k) \in \mathbf{N}^{3}$. The triple sequence space of Bernstein polynomials of $\left(B_{m n k}(f, X)\right)$ satisfying $\left(B_{m n k}(f, X)\right) \in$ $\left[\mu_{m n k}, \lambda_{m n k}\right]$, for all $m, n, k$. Then the triple sequence space of Bernstein polynomials of $\left(B_{m n k}(f, X)\right)$ may not be convergent, but the inequality 


$$
\begin{gathered}
d\left(B_{m n k}(f, X), f(X)\right) \leq d\left(B_{m n k}(f, X), B_{m n k}(f, Y)\right)+ \\
d\left(B_{m n k}(f, Y), f(Y)\right) \leq r+d\left(B_{m n k}(f, Y), f(Y)\right)
\end{gathered}
$$

implies that the triple sequence space of Bernstein polynomials of $\left(B_{m n k}(f, X)\right)$ is $r$ - convergent.

In this paper, we first define the concept of rough convergence of a triple sequence space of Bernstein polynomials of fuzzy numbers. Also obtain the relation between the set of rough limit and the extreme limit points of a triple sequence space of Bernstein polynomials of fuzzy numbers. We show that the rough limit set of a triple sequence space of Bernstein polynomials is closed, bounded and convex.

\section{Definitions and Preliminaries}

A fuzzy number $X$ is a fuzzy subset of the real $\mathbf{R}^{3}$, which is normal fuzzy convex, upper semi-continuous, and the $X^{0}$ is bounded where $X^{0} ;=$ $c l\left\{x \in \mathbf{R}^{3}: X(x)>0\right\}$ and $c l$ is the closure operator. These properties imply that for each $\alpha \in(0$,$] , the \alpha$ - level set $X^{\alpha}$ defined by

$$
X^{\alpha}=\left\{x \in \mathbf{R}^{3}: X(x) \geq \alpha\right\}=\left[\underline{X}^{\alpha}, \bar{X}^{\alpha}\right]
$$

is a non empty compact convex subset of $\mathbf{R}^{3}$.

The supremum metric $d$ on the set $L\left(\mathbf{R}^{3}\right)$ is defined by

$$
d(X, Y)=\sup _{\alpha \in[0,1]} \max \left(\left|\underline{X}^{\alpha}-\underline{Y}^{\alpha}\right|,\left|\bar{X}^{\alpha}-\bar{Y}^{\alpha}\right|\right) .
$$

Now, given $X, Y \in L\left(\mathbf{R}^{3}\right)$, we define $X \leq Y$ if $\underline{X}^{\alpha} \leq \underline{Y}^{\alpha}$ and $\bar{X}^{\alpha} \leq \bar{Y}^{\alpha}$ for each $\alpha \in[0$,$] .$

We write $X \leq Y$ if $X \leq Y$ and there exists an $\alpha_{0} \in[0,1]$ such that $\underline{X}^{\alpha_{0}} \leq \underline{Y}^{\alpha_{0}}$ or $\bar{X}^{\alpha_{0}} \leq \bar{Y}^{\alpha_{0}}$.

A subset $E$ of $L\left(\mathbf{R}^{3}\right)$ is said to be bounded above if there exists a fuzzy number $\mu$, called an upper bound of $E$, such that $X \leq \mu$ for every $X \in E$. $\mu$ is called the least upper bound of $E$ if $\mu$ is an upper bound and $\mu \leq \mu^{\prime}$ for all upper bounds $\mu^{\prime}$.

A lower bound and the greatest lower bound are defined similarly. $E$ is said to be bounded if it is both bounded above and below.

The notions of least upper bound and the greatest lower bound have been defined only for bounded sets of fuzzy numbers. If the set $E \subset L\left(\mathbf{R}^{3}\right)$ is bounded then its supremum and infimum exist. 
266 M. J. Bharathi, S. Velmurugan, N. Subramanian and R. Srikanth

The limit infimum and limit supremum of a triple sequence spaces $\left(X_{m n k}\right)$ is defined by

$$
\begin{aligned}
\lim _{m n k \rightarrow \infty} \inf X_{m n k} & :=\inf A_{X} . \\
\lim _{m n k \rightarrow \infty} \sup X_{m n k} & :=\inf B_{X} .
\end{aligned}
$$

where

$$
A_{X}:=\left\{\mu \in L\left(\mathbf{R}^{3}\right): \text { The set }\left\{(m, n, k) \in \mathbf{N}^{3}: X_{m n k}<\mu\right\} \text { is infinite }\right\}
$$
$B_{X}:=\left\{\mu \in L\left(\mathbf{R}^{3}\right):\right.$ The set $\left\{(m, n, k) \in \mathbf{N}^{3}: X_{m n k}>\mu\right\}$ is infinite $\}$. Now, given two fuzzy numbers $X, Y \in L\left(\mathbf{R}^{3}\right)$, we define their sum as $Z=X+Y$, where $\underline{Z}^{\alpha}:=\underline{X}^{\alpha}+\underline{Y}^{\alpha}$ and $\bar{Z}^{\alpha}:=\bar{X}^{\alpha}+\bar{Y}^{\alpha}$ for all $\alpha \in[0,1]$. To any real number $a \in \mathbf{R}^{3}$, we can assign a fuzzy number $a_{1} \in L\left(\mathbf{R}^{3}\right)$, which is defied by

$$
a_{1}(x)=\left\{\begin{array}{lc}
1, & \text { if } x=a \\
0, & \text { otherwise }
\end{array}\right\}
$$

An order interval in $L\left(\mathbf{R}^{3}\right)$ is defined by $[X, Y]:=\left\{Z \in L\left(\mathbf{R}^{3}\right): X \leq Z \leq Y\right\}$, where $X, Y \in L\left(\mathbf{R}^{3}\right)$.

A set $E$ of fuzzy numbers is called convex if $\lambda \mu_{1}+(1-\lambda) \mu_{2} \in E$ for all $\lambda \in[0,1]$ and $\mu_{1}, \mu_{2} \in E$.

\section{Main Results}

\subsection{Theorem}

Let $f$ be a continuous function defined on the closed interval $[0,1]$. A triple sequence of Bernstein polynomials of $\left(B_{m n k}(f, X)\right)$ of real numbers. If $f(X) \in L I M^{r} B_{m n k}(f, X)$, then $\operatorname{diam}\left(\lim \sup B_{m n k}(f, X), f(X)\right) \leq r$ and

$\operatorname{diam}\left(\lim \inf B_{m n k}(f, X), f(X)\right) \leq r$.

Proof: We assume that diam (lim sup $\left.B_{m n k}(f, X), f(X)\right)>r$.

Define $\tilde{\epsilon}:=\frac{\left(\lim \sup B_{m n k}(f, X), f(X)\right)-r}{2}$. By definition of limit supremem, we have that given $m_{\tilde{\epsilon}}^{\prime}, n_{\tilde{\epsilon}}^{\prime}, k_{\tilde{\epsilon}}^{\prime} \in \mathbf{N}^{3}$ there exists an $(m, n, k) \in \mathbf{N}^{3}$ with $m \geq$ $m_{\tilde{\epsilon}}^{\prime}, n \geq n_{\tilde{\epsilon}}^{\prime}, k \geq k_{\tilde{\epsilon}}^{\prime}$ such that diam (lim sup $\left.B_{m n k}(f, X), f(X)\right) \leq \tilde{\epsilon}$. Also, since $B_{m n k}(f, X) \rightarrow^{r} f(X)$ as $m, n, k \rightarrow \infty$, there is an integer $m_{\tilde{\epsilon}}^{\prime \prime}, n_{\tilde{\epsilon}}^{\prime \prime}, k_{\tilde{\epsilon}}^{\prime \prime}$ so that 


$$
d\left(B_{m n k}(f, X), f(X)\right)<r+\tilde{\epsilon}
$$

whenever $m \geq m_{\tilde{\epsilon}}^{\prime \prime}, n \geq n_{\tilde{\epsilon}}^{\prime \prime}, k \geq k_{\tilde{\epsilon}}^{\prime \prime}$. Let

$$
\left(m_{\tilde{\epsilon}}, n_{\tilde{\epsilon}}, k_{\tilde{\epsilon}}\right):=\max \left\{\left(m_{\tilde{\epsilon}}^{\prime}, n_{\tilde{\epsilon}}^{\prime}, k_{\tilde{\epsilon}}^{\prime}\right),\left(m_{\tilde{\epsilon}}^{\prime \prime}, n_{\tilde{\epsilon}}^{\prime \prime}, k_{\tilde{\epsilon}}^{\prime \prime}\right)\right\} .
$$

There exists $(m, n, k) \in \mathbf{N}^{3}$ such that $m \geq m_{\tilde{\epsilon}}, n \geq n_{\tilde{\epsilon}}, k \geq k_{\tilde{\epsilon}}$ and $\operatorname{diam}\left(\lim \sup B_{m n k}(f, X), f(X)\right) \leq(f, X) \operatorname{diam}\left(\lim \sup B_{m n k}(f, X), B_{m n k}(f, X)\right)+$ $\operatorname{diam}\left(B_{m n k}, f(X)\right)$

$$
\begin{aligned}
& <\tilde{\epsilon}+r+\tilde{\epsilon} \\
& <r+2 \tilde{\epsilon} \\
& =r+\operatorname{diam}\left(\lim \sup B_{m n k}(f, X), f(X)\right)-r \\
& =\operatorname{diam}\left(\lim \sup B_{m n k}(f, X), f(X)\right) .
\end{aligned}
$$

The contradiction proves the theorem. Similarly, diam (lim inf $\left.B_{m n k}(f, X), f(X)\right) \leq$ $r$ can be proved using definition of limit infimum.

\subsection{Theorem}

Let $f$ be a continuous function defined on the closed interval $[0,1]$. A triple sequence of Bernstein polynomials of $\left(B_{m n k}(f, X)\right)$ of real numbers. If $L I M^{r} B_{m n k}(f, X) \neq \phi$, then we have $L I M^{r} B_{m n k}(f, X) \subseteq\left[\left(\limsup B_{m n k}(f, X)\right)-r_{1},\left(\liminf B_{m n k}(f, X)\right)+r_{1}\right]$.

Proof: To prove that $f(X) \in\left[\left(\limsup B_{m n k}(f, X)\right)-r_{1},\left(\liminf B_{m n k}(f, X)\right)+r_{1}\right]$ for an arbitrary $f(X) \in L I M^{r} B_{m n k}(f, X)$, i.e.,

$$
\left(\limsup B_{m n k}(f, X)\right)-r_{1} \leq f(X) \leq\left(\liminf B_{m n k}(f, X)\right)+r_{1} .
$$

Let us assume that $\left(\limsup B_{m n k}(f, X)\right)-r_{1} \leq f(X)$ does not hold.Thus, there exists an $\alpha_{0} \in[0,1]$ such that

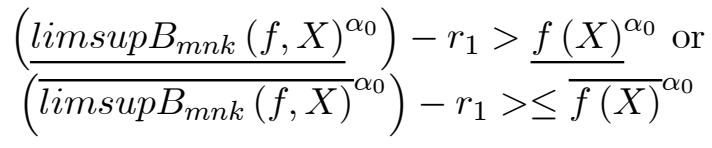

holds i.e.,

$$
\begin{aligned}
& \left.\left(\underline{\limsup }_{m n k}(f, X)\right)^{\alpha_{0}}\right)-\underline{f(X)}^{\alpha_{0}}>r_{1} \text { or } \\
& \left({\overline{\limsup B_{m n k}(f, X)}}^{\alpha_{0}}\right)-\overline{\leq \overline{f(X)}}^{\alpha_{0}}>r_{1} \text {. }
\end{aligned}
$$


268 M. J. Bharathi, S. Velmurugan, N. Subramanian and R. Srikanth

On the other hand, by theorem (3.1) we have

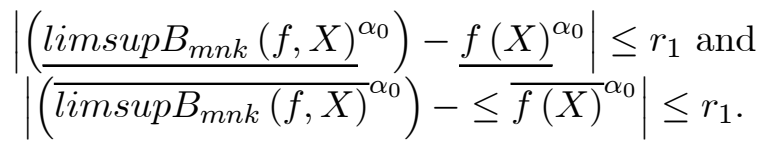

We obtain a contradiction. Hence we get $\left(\limsup B_{m n k}(f, X)\right)-r_{1} \leq$ $f(X)$. By using the similar arguments and get it for second part.

\subsection{Note}

The converse inclusion in this theorem holds for $f$ be a continuous function defined on the closed interval $[0,1]$. A triple sequence of Bernstein polynomials of $\left(B_{m n k}(f, X)\right)$ of real numbers, but it may not hold for triple sequences of Bernstein polynomials of fuzzy numbers as in the following example:

\section{Example: Define}

$$
B_{m n k}(f, X)=\left\{\begin{array}{cc}
\frac{-1}{2(m n k)} X+1, & \text { if } X \in[0,1], \\
0, & \text { otherwise }
\end{array}\right\}
$$

and

$$
f(X)=\left\{\begin{array}{cc}
1, & \text { if } X \in[0,1], \\
0, & \text { otherwise }
\end{array}\right\} .
$$

Then we have $\left|\overline{f(X)}^{1}-{\overline{B_{m n k}(f, X)}}^{1}\right|=|1-0|=1$, i.e., $d\left(B_{m n k}(f, X), f(X)\right) \geq$ 1 for all $(m, n, k) \in \mathbf{N}^{3}$. Although the triple sequence spaces of Bernstein poynomials of $\left(B_{m n k}(f, X)\right)$ is not convergent to $f(X), \limsup B_{m n k}(f, X)$ and $\operatorname{limin} f B_{m n k}(f, X)$ of this triple sequence space of Bernstein polynomials are equal to $f(X)$. Hence we get

$$
\begin{gathered}
L \in\left[\limsup B_{m n k}(f, X)-\left(\frac{1}{2}\right)_{1}, \liminf B_{m n k}(f, X)+\left(\frac{1}{2}\right)_{1}\right], \text { but } \\
f(X) \notin L I M^{\frac{1}{2}} B_{m n k}(f, X) .
\end{gathered}
$$

\subsection{Theorem}

Let $f$ be a continuous function defined on the closed interval $[0,1]$. A triple sequence of Bernstein polynomials of $\left(B_{m n k}(f, X)\right)$ of real numbers converges to the fuzzy number $f(X)$, then 


$$
L I M^{r} B_{m n k}(f, X)=\bar{B}_{r}(f(X)):=\left\{\mu \in f(X)\left(\mathbf{R}^{3}\right): d(\mu, f(X)) \leq r\right\}
$$

Proof: Let $\epsilon>0$. Since the triple sequence space of Bernstein polynomials of $\left(B_{m n k}(f, X)\right)$ is convergent to $f(X)$, there is an integer $m_{\epsilon}, n_{\epsilon}, k_{\epsilon}$ so that

$$
d\left(B_{m n k}(f, X), f(X)\right)<\epsilon \text { whenever } m \geq m_{\epsilon}, n \geq n_{\epsilon}, k \geq k_{\epsilon} .
$$

Let $Y \in \bar{B}_{r}(f(X))$, we have

$$
\begin{gathered}
d\left(B_{m n k}(f, X), Y\right) \leq d\left(B_{m n k}(f, X), f(X)\right)+d(f(X), Y)<\epsilon+r \text { for } \\
\text { every } m \geq m_{\epsilon}, n \geq n_{\epsilon}, k \geq k_{\epsilon} .
\end{gathered}
$$

Hence we have $Y \in L I M^{r} B_{m n k}(f, X)$.

Now let $Y \in L I M^{r} B_{m n k}(f, X)$. Hence there is an integer $m_{\epsilon}^{\prime}, n_{\epsilon}^{\prime}, k_{\epsilon}^{\prime}$ so that

$$
d\left(B_{m n k}(f, X), Y\right)<r+\epsilon
$$

whenever $m \geq m_{\epsilon}^{\prime}, n \geq n_{\epsilon}^{\prime}, k \geq k_{\epsilon}^{\prime}$. Let

$$
\left(m_{\epsilon}^{\prime \prime}, n_{\epsilon}^{\prime \prime}, k_{\epsilon}^{\prime \prime}\right):=\max \left\{\left(m_{\epsilon}, n_{\epsilon}, k_{\epsilon}\right),\left(m_{\epsilon}^{\prime}, n_{\epsilon}^{\prime}, k_{\epsilon}^{\prime}\right)\right\}
$$

for all $m \geq m_{\epsilon}^{\prime \prime}, n \geq n_{\epsilon}^{\prime \prime}, k \geq k_{\epsilon}^{\prime \prime}$, we obtain

$$
\begin{gathered}
d(Y, f(X)) \leq d\left(Y, B_{m n k}(f, X)\right)+d\left(B_{m n k}(f, X), f(X)\right)<r+\epsilon+\epsilon< \\
r+2 \epsilon .
\end{gathered}
$$

Since $\epsilon$ is arbitrary, we have $d(Y, f(X)) \leq r$. Hence we get $Y \in$ $\bar{B}_{r}(f(X))$. Thus, if the triple sequence space of Bernstein polynomials of $\left(B_{m n k}(f, X)\right) \rightarrow^{r} f(X)$, then $L I M^{r} B_{m n k}(f, X)=\bar{B}_{r}(f(X))$.

\subsection{Theorem}

Let $f$ be a continuous function defined on the closed interval $[0,1]$. A triple sequence of real numbers of Bernstein polynomials of $\left(B_{m n k}(f, X)\right)$ and $\left(B_{m n k}(f, Y)\right) \in f(X)\left(\mathbf{R}^{3}\right)$. If $B_{m n k}(f, X) \rightarrow^{r} f(X)$ then $B_{m n k}(f, Y) \rightarrow^{r}$ $f(Y)$ and $d\left(B_{m n k}(f, X), B_{m n k}(f, Y)\right) \leq r$ for every $(m, n, k) \in \mathbf{N}^{3}$.

Proof: Assume that $B_{m n k}(f, Y) \rightarrow^{r} f(Y)$, as $m, n, k \rightarrow \infty$ and $d\left(B_{m n k}(f, X), B_{m n k}(f, Y)\right) \leq r$ for every $(m, n, k) \in \mathbf{N}^{3}$. We have $B_{m n k}(f, Y) \rightarrow^{r} f(Y)$, as $m, n, k \rightarrow \infty$ means that for every $\epsilon>0$ there exists an $m_{\epsilon}, n_{\epsilon}, k_{\epsilon}$ such that 
270 M. J. Bharathi, S. Velmurugan, N. Subramanian and R. Srikanth

$$
d\left(B_{m n k}(f, Y), f(Y)\right)<\epsilon \text { for all } m \geq m_{\epsilon}, n \geq n_{\epsilon}, k \geq k_{\epsilon} .
$$

If the in equality $d\left(B_{m n k}(f, X), B_{m n k}(f, Y)\right) \leq r$ yields then

$$
\begin{gathered}
d\left(B_{m n k}(f, X), f(X)\right) \leq \\
d\left(B_{m n k}(f, X), B_{m n k}(f, Y)\right)+d\left(B_{m n k}(f, Y), f(Y)\right)<r+\epsilon \text { for all } \\
m \geq m_{\epsilon}, n \geq n_{\epsilon}, k \geq k_{\epsilon} .
\end{gathered}
$$

Hence the triple sequence space of Bernstein polynomials of $\left(B_{m n k}(f, X)\right)$ is $r$ - convergent to the fuzzy number $f(X)$.

\subsection{Theorem}

Let $f$ be a continuous function defined on the closed interval $[0,1]$. A triple sequence of Bernstein polynomials of $\left(B_{m n k}(f, X)\right)$ of real numbers and the diameter of an $r$ - limit set is not greater than $3 r$.

Proof: We have to prove that

$$
\sup \left\{d(W, Z): W, Y, Z \in L I M^{r} B_{m n k}(f, X)\right\} \leq 3 r .
$$

Assume on the contrary that

$$
\sup \left\{d(W, Z): W, Y, Z \in L I M^{r} B_{m n k}(f, X)\right\}>3 r .
$$

By this assumption, there exists, $W, Y, Z \in L I M^{r} B_{m n k}(f, X)$ satisfying $\lambda:=d(W, Z)>3 r$. For an arbitrary $\epsilon \in\left(0, \frac{\lambda}{3}-r\right)$, we have $\exists\left(m_{\epsilon}^{\prime}, n_{\epsilon}^{\prime}, k_{\epsilon}^{\prime}\right) \in \mathbf{N}^{3}: \forall(m, n, k) \geq\left(m_{\epsilon}^{\prime}, n_{\epsilon}^{\prime}, k_{\epsilon}^{\prime},\right) \Longrightarrow d\left(B_{m n k}(f, X), W\right) \leq$ $r+\epsilon$,

$\exists\left(m_{\epsilon}^{\prime \prime}, n_{\epsilon}^{\prime \prime}, k_{\epsilon}^{\prime \prime}\right) \in \mathbf{N}^{3}: \forall(m, n, k) \geq\left(m_{\epsilon}^{\prime \prime}, n_{\epsilon}^{\prime \prime}, k_{\epsilon}^{\prime \prime},\right) \Longrightarrow d\left(B_{m n k}(f, X), Y\right) \leq$ $r+\epsilon$,

$\exists\left(m_{\epsilon}^{\prime \prime \prime}, n_{\epsilon}^{\prime \prime \prime}, k_{\epsilon}^{\prime \prime \prime}\right) \in \mathbf{N}^{3}: \forall(m, n, k) \geq\left(m_{\epsilon}^{\prime \prime \prime}, n_{\epsilon}^{\prime \prime \prime}, k_{\epsilon}^{\prime \prime \prime},\right) \Longrightarrow d\left(B_{m n k}(f, X), Z\right) \leq$ $r+\epsilon$.

Define $\left(m_{\epsilon}, n_{\epsilon}, k_{\epsilon}\right):=\max \left\{\left(m_{\epsilon}^{\prime}, n_{\epsilon}^{\prime}, k_{\epsilon}^{\prime}\right),\left(m_{\epsilon}^{\prime \prime}, n_{\epsilon}^{\prime \prime}, k_{\epsilon}^{\prime \prime}\right),\left(m_{\epsilon}^{\prime \prime \prime}, n_{\epsilon}^{\prime \prime \prime}, k_{\epsilon}^{\prime \prime \prime}\right)\right\}$.

Thus we get

$$
\begin{aligned}
d(W, Z) \leq d\left(B_{m n k}(f, X)\right. & , W)+d\left(B_{m n k}(f, X), Y\right)+d\left(B_{m n k}(f, X), Z\right) \\
& <(r+\epsilon)+(r+\epsilon)+(r+\epsilon) \\
& <3(r+\epsilon) \\
& <3 r+3\left(\frac{\lambda}{3}-r\right)<3 r+\lambda-3 r \\
& =\lambda \text { for all } m \geq m_{\epsilon}, n \geq n_{\epsilon}, k \geq k_{\epsilon}
\end{aligned}
$$

which contradicts to the fact that $\lambda=d(W, Z)$. 


\subsection{Theorem}

Let $f$ be a continuous function defined on the closed interval $[0,1]$. A triple sequence of Bernstein polynomials of $\left(B_{m n k}(f, X)\right)$ of real numbers is analytic if and only if there exists an $r \geq 0$ such that $L I M^{r} B_{m n k}(f, X) \neq \phi$.

Proof: Necessity: Let the triple sequence space of Bernstein polynomials of $\left(B_{m n k}(f, X)\right)$ be a analytic sequence and

$$
s:=\sup \left\{d\left(B_{m n k}(f, X)^{1 / m+n+k}, 0\right):(m, n, k) \in \mathbf{N}^{3}\right\}<\infty .
$$

Then we have $0 \in L I M^{s} B_{m n k}(f, X)$, i.e., $L I M^{r} B_{m n k}(f, X) \neq \phi$, where $r=s$.

Sufficiency: If $L I M^{r} B_{m n k}(f, X) \neq \phi$ for some $r \geq 0$, then there exists $f(X) \in L I M^{r} B_{m n k}(f, X)$. By definition, for every $\epsilon>0$ there is an integer $\left(m_{\epsilon}, n_{\epsilon}, k_{\epsilon}\right)$ so that

$$
d\left(B_{m n k}(f, X), f(X)\right)<r+\epsilon \text { whenever } m \geq m_{\epsilon}, n \geq n_{\epsilon}, k \geq k_{\epsilon} .
$$

Define

$$
\begin{gathered}
t=t(\epsilon):= \\
\operatorname{Max}\left\{d(f(X), 0), d\left(B_{111}(f, X), 0\right), \cdots, d\left(B_{m_{\epsilon} n_{\epsilon} k_{\epsilon}}(f, X), 0\right), r+\epsilon\right\} .
\end{gathered}
$$

Then we have

$$
B_{m n k} \in\left\{\mu \in f(X)\left(\mathbf{R}^{3}\right): d(\mu, 0) \leq t+r+\epsilon\right\} \text { for every }(m, n, k) \in \mathbf{N}^{3},
$$

which proves the boundedness of the triple sequence space of Bernstein polynomials of $\left(B_{m n k}(f, X)\right)$.

\subsection{Theorem}

Let $f$ be a continuous function defined on the closed interval $[0,1]$. A triple sequence of Bernstein polynomials of $\left(B_{u_{m} v_{n} w_{k}}(f, X)\right)$ of real numbers is a sub sequence of a triple sequence space of Bernstein polynomials of $\left(B_{m n k}(f, X)\right)$, then $L I M^{r} B_{m n k}(f, X) \subset L I M^{r} B_{u_{m} v_{n} w_{k}}(f, X)$.

Proof: Omitted. 


\subsection{Theorem}

Let $f$ be a continuous function defined on the closed interval $[0,1]$. A triple sequence of Bernstein polynomials of $\left(B_{m n k}(f, X)\right)$ of real numbers, for all $r \geq 0$, the $r$-limit set $L I M^{r} B_{m n k}(f, X)$ of an arbitrary triple sequence space of Bernstein polynomials of $B_{m n k}(f, X)$ is closed.

Proof: Let $\left(Y_{m n k}\right) \subset L I M^{r} B_{m n k}(f, Y)$ and $B_{m n k}(f, Y) \rightarrow f(Y)$ as $m, n, k \rightarrow \infty$. Let $\epsilon>0$. Since the triple sequence space of Bernstein polynomials of $\left(B_{m n k}(f, Y)\right) \rightarrow^{r} f(Y)$, there is an integer $i_{\epsilon} j_{\epsilon} \ell_{\epsilon}$ so that

$$
d\left(B_{m n k}(f, Y), f(Y)\right)<\frac{\epsilon}{2} \text { whenever } m \geq i_{\epsilon}, n \geq j_{\epsilon}, k \geq \ell_{\epsilon} .
$$

Since $B_{i_{\epsilon} j_{\epsilon} \ell_{\epsilon}}(f, Y) \in L I M^{r} B_{m n k}(f, X)$, there is an integer $\left(m_{\epsilon} n_{\epsilon} k_{\epsilon}\right)$ so that

$$
d\left(B_{m n k}(f, X), B_{i_{\epsilon} j_{\epsilon} \ell_{\epsilon}}(f, Y)\right)<r+\frac{\epsilon}{2} \text { whenever } m \geq m_{\epsilon}, n \geq n_{\epsilon}, k \geq k_{\epsilon} .
$$

Therefore, we have

$$
\begin{gathered}
d\left(B_{m n k}(f, X), f(X)\right) \leq d\left(B_{m n k}(f, X), B_{i_{\epsilon} j_{\epsilon} \ell_{\epsilon}}(f, Y)\right)<r+\frac{\epsilon}{2}+\frac{\epsilon}{2}=r+\epsilon \\
\text { for every } m \geq m_{\epsilon}, n \geq n_{\epsilon}, k \geq k_{\epsilon} .
\end{gathered}
$$

Hence $L \in L I M^{r} B_{m n k}(f, X)$ implies that the set $L I M^{r} B_{m n k}(f, X)$ is closed.

\section{Conclusions and Future Work}

We introduced triple sequence space of Bernstein polynomials of rough convergence of fuzzy numbers. For the reference sections, consider the following introduction described the main results are motivating the research.

Competing Interests: The authors declare that there is not any conflict of interests regarding the publication of this manuscript.

\section{Acknowledgement}

The authors are extremely grateful to the anonymous learned referee(s) for their keen reading, valuable suggestion and constructive comments for the improvement of the manuscript. The authors are thankful to the editor(s) and reviewers of Proyecciones Journal of Mathematics. 


\section{References}

[1] S. Aytar, "Rough statistical convergence", Numerical functional analysis and optimization, vol. 29, no. 3-4, pp. 291-303, Apr. 2008, doi: $10.1080 / 01630560802001064$.

[2] S. Aytar, "The rough limit set and the core of a real sequence", Numerical functional analysis and optimization, vol. 29, no. 3-4, pp. 283-290, May 2008, doi: $10.1080 / 01630560802001056$

[3] A. Esi, "On some triple almost lacunary sequence spaces defined by Orlicz functions", Research and reviews: discrete mathematical structures, vol. 1, no. 2, pp. 16-25, 2014. [On line]. Available: https://bit.ly/3bzXom4

[4] A. Esi and M. N. Catalbas, "Almost convergence of triple sequences", Global journal of mathematical analysis, vol. 2, no. 1, 2014, doi: 10.14419/gjma.v2i1.1709

[5] A. Esi and E. Savas, "On lacunary statistically convergent triple sequences in probabilistic normed space", Applied mathematics \& information sciences, vol. 9, no. 5, pp. 2529-2534, Sep. 2015. [On line]. Available: https://bit.ly/2yF0aHW

[6] A. Esi, S. Araci, and M. Acikgoz, "Statistical convergence of Bernstein operators", Applied mathematics \& information sciences, vol. 10, no. 6, pp. 2083-2086, Dec. 2016. [On line]. Available: https://bit.ly/34UJVTt

[7] A. Esi, S. Araci, and Ayten Esi, " $\lambda$-Statistical convergence of Bernstein polynomial sequences", Advances and applications in mathematical sciences, vol. 16, no. 3, pp. 113-119, Jan. 2017. [On line]. Available: https://bit.ly/2XVlbsz

[8] A. Esi, N. Subramanian, and A. Esi, "On Triple sequence space of Bernstein operator of Rough I- convergence pre-cauchy sequences", Proyecciones (Antofagasta. On line), vol. 36, no. 4, pp. 567-587, 2017, doi: 10.4067/S071609172017000400567.

[9] A. J. Datta, A. Esi, and B. C. Tripathy, "Statistically convergent triple sequence spaces defined by Orlicz function", Journal of mathematical analysis, vol. 4, no. 2, pp. 16-22, 2013. [On line]. Available: https://bit.ly/353IulB

[10] S. Debnath, B. Sarma, and B. C. Das, "Some generalized triple sequence spaces of real numbers", Journal of nonlinear analysis and optimization, vol. 6, no. 1, pp. 71-79, 2015. [On line]. Available: https://bit.ly/2VsTxBC

[11] E. Dündar and C. Cakan, "Rough I- convergence", Demonstratio mathematica, Accepted.

[12] H. X. Phu, "Rough convergence in normed linear spaces", Numerical functional analysis and optimization, vol. 22, no. 1-2, pp. 199-222, Mar. 2001, doi: 10.1081/NFA-100103794.

[13] H.X. Phu, "Rough continuity of linear operators", Numerical functional analysis and optimization, vol. 23, no. 1-2, pp. 139-146, Jan. 2002, doi: 10.1081/NFA120003675. 
274 M. J. Bharathi, S. Velmurugan, N. Subramanian and R. Srikanth

[14] H.X. Phu, "Rough convergence in infinite dimensional normed spaces", Numerical functional analysis and optimization, vol. 24, no. 3-4, pp. 285-301, Jan. 2003, doi: 10.1081/NFA-120022923.

[15] A. Sahiner, M. Gurdal, and F. K. Duden, "Triple sequences and their statistical convergence", Selçuk journal of applied mathematics, vol. 8, no. 2, pp. 49-55, 2007. [On line]. Available: https://bit.ly/2VvgnZy

[16] A. Sahiner, B. C. Tripathy, "Some I related properties of triple sequences", Selçuk journal of applied mathematics, vol. 9, no. 2, pp. 9-18, 2008. [On line]. Available: https://bit.ly/3cDBpdX

[17] N. Subramanian and A. Esi, "The generalized tripled difference of $\chi 3$ sequence spaces", Global journal of mathematical analysis, vol. 3, no. 2, pp. 54-60, Mar. 2015, doi: 10.14419/gjma.v3i2.4412. 